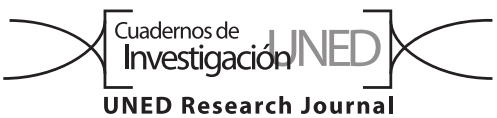

\title{
NOTA
}

\section{El mono Cebus imitator (Primates: Cebidae) se alimenta del fruto de Hedyosmum mexicanum (Chloranthaceae) en las alturas de Costa Rica}

\author{
Pablo Marín Pacheco \\ Universidad Técnica Nacional, Ingeniería en Manejo Forestal y Vida Silvestre, Atenas, Costa Rica; pomarin@est.utn.ac.cr \\ Recibido 19-X-2018 • Corregido 31-XII-2018 • Aceptado 30-I-2019
}

\begin{abstract}
The monkey Cebus imitator (Primates: Cebidae) feeds on fruits of Hedyosmum mexicanum (Chloranthaceae) in Costa Rica highlands". In neotropical forests, the feeding habits of the white-faced monkey are widely known. However, little information is available regarding their diet and behavior in high-elevation tropical forests. Here I document (by photos) the consumption of the fruit of Hedyosmum mexicanum by a troop of Cebus imitator, on the slopes of Barva Volcano, Costa Rica, $2625 \mathrm{~m}$ above sea level.
\end{abstract}

Key words: Cebus imitator, Cebidae, white-faced monkey, feeding, Hedyosmum mexicanum, Costa Rica.
RESUMEN: En los bosques neotropicales se conoce con amplitud los hábitos alimenticios del mono carablanca. Sin embargo, en los ecosistemas tropicales de altas elevaciones, se dispone de poca información respecto a su dieta y comportamiento. Motivo, por el cual, presento con evidencias (fotografías) el primer reporte del consumo del fruto Hedyosmum mexicanum por parte de una tropa de Cebus imitator, en las faldas del Volcán Barva, Costa Rica, $2625 \mathrm{~m}$ sobre el nivel del mar.

Palabras claves. Cebus imitator, Cebidae, mono carablanca, alimentación, Hedyosmum mexicanum, Costa Rica.
El mono carablanca (Cebus imitator) es la especie del orden primates que presenta el mayor número de ejemplares en Costa Rica, destacando por ser el género de mayor distribución en todo América Latina (Chinchilla, Guerrero, Gutiérrez, Sánchez, \& Idalia, 2007; Madrigal \& Gonzáles, 2016). Esta especie se distribuye desde el noreste de Honduras hasta el sur de Costa Rica y habitualmente pueden ser encontrados en los bosques maduros, bosques secundarios, pantanos, manglares, bosques de galería, hasta los 3000 metros sobre el nivel del mar (Wainwright, 2007; Crofoot \& Tórrez, 2013; Rodríguez, Ramírez, Villalobos, \& Sánchez, 2014). El mono cariblanco es una especie de comportamiento diurno, arborícola y gregarias, donde suelen conformar tropas de 5 a 36 individuos (Carrillo, Wong, \& Sáenz, 2002).

El Cebus imitator juega un papel importante dentro del ecosistema, debido a que son participes de la dispersión de semillas, contribuyendo de esta manera con la regeneración de los bosques del neotrópico (Fedigan, Fedigan, \& Chapman, 1985). Como prueba de ello existen reportes de individuos de C. imitator dispersando semillas intactas de 67 especies en un estudio realizado en Panamá (Wehncke, Hubbell, Fosters, \& Dalling, 2003).
El tipo de hábito alimenticio del mono carablanca es omnívoro y su dieta se basa principalmente de frutas, néctar, flores, huevos de aves, insectos y vertebrados como ratones, murciélagos, pequeños reptiles, anfibios, aves, ardillas y crías de coatis (Fedigan 1990; Sáenz \& Sáenz, 2008). La base de la dieta de C. imitator son las frutas, según un estudio realizado en el Parque Nacional Santa Rosa, en el cual se determinó que el $81,2 \%$ del tiempo destinado para alimentarse lo pasan consumiendo frutos (Fedigan et al., 1985).

El presente manuscrito, hace referencia al primer registro de consumo del fruto de la especie arbórea Hedyosmum mexicanum por parte del C. imitator. El día 25 de junio del 2017, sobre las 10:45am, en las faldas del Parque Nacional Braulio Carrillo, Sector Volcán Barva, en las coordenadas $10^{\circ} 7^{\prime} 44,69^{\prime \prime} \mathrm{N} \& 84^{\circ} 7^{\prime} 32,31^{\prime \prime} \mathrm{W}$, a los 2625 $m$ de elevación, se registró una tropa aproximadamente de 5 a 7 individuos, del cual tres de ellos se observaron consumiendo los frutos del árbol Hedyosmum mexicanum, durante un lapso de 10-15min. aproximadamente (Fig. 1).

En el neotrópico, a través de la investigación, se conoce de una numerosa lista de frutos comestibles por parte 


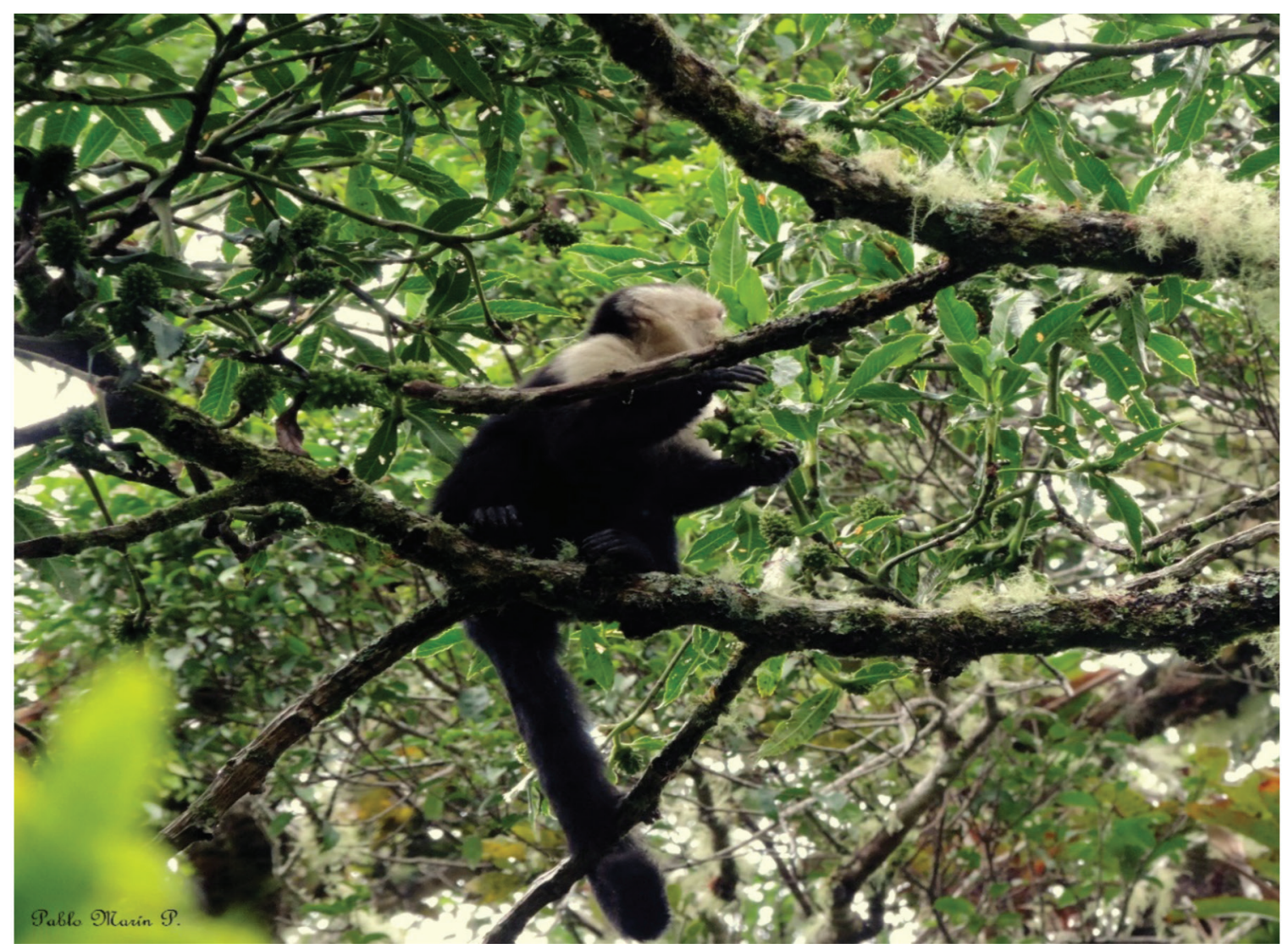

Fig. 1. Individuo de Cebus imitator consumiendo los frutos de la especie arbórea Hedyosmum mexicanum, en las faldas del Volcán Barva, Costa Rica.

de los monos carablanca (Freese, 1977). Sin embargo, se desconocía en su totalidad que el fruto de $H$. mexicanum fuera parte de la dieta del C. imitator. Confirmándose de esta manera, como la primera especie del género Cebus, en consumir estos frutos.

Por otro lado, cabe destacar la importancia de la especie $H$. mexicanum para las poblaciones de los monos carablanca de los ecosistemas de altas elevaciones. Por lo cual, es importante tomar en consideración el uso del árbol $H$. mexicanum en la inclusión de especies para los planes de restauración ecológica en sitios aledaños.

\section{Ética, conflicto de intereses y declaración de finan-} ciamiento: el autor declara haber cumplido con todos los requisitos éticos y legales pertinentes, tanto durante el estudio como en el manuscrito; que no hay conflictos de interés de ningún tipo, y que todas las fuentes financieras se detallan plena y claramente en la sección de agradecimientos. Asimismo, está de acuerdo con la versión editada final del documento. El respectivo documento legal firmado se encuentra en los archivos de la revista.

\section{REFERENCIAS}

Carrillo, E., Wong, G., \& Sáenz, J. (2002). Mamíferos de Costa Rica. Santo Domingo, Heredia, Costa Rica: Instituto Nacional de Biodiversidad (INBio).

Chinchilla, M., Guerrero, O., Gutiérrez, G., Sánchez, R., \& Idalia, B. (2007). Parásitos en monos carablanca Cebus capucinus (Primates: Cebidae) de Costa Rica. Parasitología latinoamericana, 62(3-4), 170-175. DOI: 10.4067/ S0717-77122007000200011

Crofoot, L., \& Tórrez, L. (2013). Cebus capucinus, ¿Por qué usan poco las zonas compartidas? Instituto Smithsonian de Investigaciones Tropicales, 8, 169-177.

Fedigan, L.M. (1990). Vertebrate predators in Cebus capucinus: meat eating a neotropical monkey. Folia Primatologica, 54(3-4),196-205. DOI: 10.1159/000156444 
Fedigan, L., Fedigan, L., \& Chapman, C. (1985). A census of Alouatta palliata and Cebus capucinus monkeys in Santa Rosa National Park, Costa Rica. Brenesia, 23, 309-322.

Freese, C. (1977). Food habits of white-faced Capuchins Cebus capucinus I. (Primates:Cebidae) in Santa Rosa National Park, Costa Rica. Brenesia, 10/11, 43-56.

Madrigal, L., \& Gonzáles, R. (Eds.). (2016). Introducción a la antropología biológica. Estados Unidos: Asociación Latinoamericana de Antropología Biológica.

Rodríguez, B., Ramírez, J., Villalobos, D., \& Sánchez, R. (2014). Actualización de la lista de especies de mamíferos vivientes de Costa Rica. Mastozoología Neotropical, 21(2), 275-289.
Sáenz, J., \& Sáenz, P. (2008). Influencia de las variables de hábitat y paisaje. Sobre la presencia-ausencia del mono tití y del mono carablanca en un área fragmentada del pacífico central, de Costa Rica. En J. Sáenz \& C. Harvey (Eds.), Evaluación y conservación de biodiversidad en paisajes fragmentados de Mesoamérica (pp. 511-545). Santo Domingo de Heredia, Costa Rica: Instituto Nacional de Biodiversidad (INBio).

Wainwright, M. (2007). The mammals of Costa Rica a natural history and field guide. New York, USA: Zona Tropical.

Wehncke, E., Hubbell, P., Fosters, B., \& Dalling, W. (2003). Seed dispersal patterns produced by white-faced monkeys: implications for the dispersal limitation of neotropical tree species. Journal of Ecology, 91(4), 677-685. DOI: 10.1046/j.1365-2745.2003.00798.x 